\title{
Peptides in the presence of aqueous ionic liquids: tunable co-solutes as denaturants or protectants? $\dagger$
}

Cite this: Phys. Chem. Chem. Phys., 2015, 17, 26049

Received 2nd July 2015,

Accepted 3rd September 2015

\author{
Volker Lesch, ${ }^{a}$ Andreas Heuer, ${ }^{a}$ Vasileios A. Tatsis, ${ }^{a}$ Christian Holm ${ }^{b}$ and \\ Jens Smiatek*b
}

DOI: $10.1039 / c 5$ cp03838c

www.rsc.org/pccp

\begin{abstract}
We studied the stability of a small $\beta$-hairpin peptide under the influence of an aqueous 1-ethyl-3-methylimidazolium acetate $\left([\mathrm{EMIM}]^{+}[\mathrm{ACE}]^{-}\right.$) solution via all-atom molecular dynamics simulations in combination with metadynamics. Our free energy results indicate a denaturation of the peptide structure in the presence of the ionic liquid which is validated by a significant broadening of the end-to-end distance. The radial distribution functions between the ions and the peptide were used for the calculation of the preferential binding coefficients in terms of the Kirkwood-Buff theory. A significant structure dependent binding behavior of acetate to the peptide was found which can be interpreted as the main reason for the denaturation of the native conformation. The outcomes of our simulations allow us to propose a simple mechanism to explain the unfolding of the peptide with regard to the specific properties of ionic liquids. Our results are in good agreement with experimental findings and demonstrate the benefits of ionic liquids as tunable co-solutes with regard to their influence on protein structural properties.
\end{abstract}

The influence of chaotropic (protein structure denaturants) and kosmotropic (protein structure protectants) co-solutes on the stability of proteins was often studied by experimental and computational approaches. ${ }^{1-8}$ It was found that molecules like urea and guanidinium lead to a destabilization of protein structures (denaturants) whereas trimethylamine- $N$-oxide (TMAO) and hydroxyectoine are known as protein protectants. ${ }^{9-11}$ A well established framework to qualitatively discuss the underlying mechanisms for denaturants and protectants was introduced by Collins et al. ${ }^{5-7}$ In this context, it was proposed that denaturants directly interact with protein surfaces whereas protectants are located in the second or the third hydration shell. Thus, the resulting behavior can be either described by a preferential binding mechanism for denaturants or a preferential exclusion

\footnotetext{
${ }^{a}$ Institut für Physikalische Chemie, Westfälische Wilhelms-Universität Münster, Corrensstrasse 28/30, D-48149 Münster, Germany

${ }^{b}$ Institut für Computerphysik, Universität Stuttgart, Allmandring 3, D-70569 Stuttgart, Germany.E-mail: smiatek@icp.uni-stuttgart.de

$\dagger$ Electronic supplementary information (ESI) available. See DOI: 10.1039/c5cp03838c
}

mechanism for protectants. Indeed, this description has been often revealed as being too general. A recent publication ${ }^{12}$ has demonstrated that the chemical properties of the protein or the surface also impose a significant contribution to the resulting preferential binding or preferential exclusion behavior.

In recent years, a class of molten salts, so-called roomtemperature ionic liquids (ILs), in addition to usual co-solutes, has been discussed as potential candidates to modify the structural properties of proteins. ${ }^{13,14}$ In fact, room-temperature ionic liquids have also attracted a lot of interest as 'designer solvents'. ${ }^{13,15-21}$ Notably, there exist a large number of ionic liquids that differ in their anion and cation composition and also in their functionalities. ${ }^{22,23}$ With regard to their influence on enzymes and proteins, ILs have been proven to have a strong impact on biocatalytic reactivity and structural stability. ${ }^{14,24-28}$ It was discussed that either denaturant or protectant behavior can be observed for differently combined ILs and proteins. Computer simulations validated that the stabilization of the enzyme cutinase significantly depends on the ionic liquid concentration of the aqueous solution. ${ }^{14}$ Pronounced stabilization effects were also observed in the presence of protic ionic liquids. It was assumed that intermolecular hydrogen bonds between the protein and the ILs are able to maintain the secondary structure even above critical temperatures. ${ }^{29,30}$ In contrast to these studies, aprotic ionic liquids with alkyl imidazolium cations have been shown to impose a denaturation effect on ribonuclease A. ${ }^{31,32}$ In three recent reviews, ${ }^{28,32,33}$ the results of several studies have been summarized which discuss Hofmeister effects ${ }^{34}$ as being one of the main reasons for IL anion- and cation related specific stabilizing and destabilizing effects.

In this communication, we study the influence of 1-ethyl-3methylimidazolium acetate $\left([\mathrm{EMIM}]^{+}[\mathrm{ACE}]^{-}\right.$, in the following denoted as EMIM and ACE) on the stability of a small $\beta$-hairpin peptide (the C-terminal from the B1 domain of the protein $\mathrm{G}$ with residues 41-56, denoted by its PDB identifier 2GB1) by atomistic molecular dynamics simulations. In addition to the computation of the Kirkwood-Buff integrals for the study of the ionic binding behavior, we have also conducted metadynamics 
simulations to evaluate the resulting free energy landscapes for the peptide in pure water and in an aqueous EMIM ACE solution. Our results evidence a denaturation effect in the presence of the ionic liquid which is mostly driven by a structure-dependent binding behavior of the acetate anions and an attraction of EMIM cations to the peptide due to short-ranged Lennard-Jones interactions. Our results are in good agreement with experimental findings for alkylimidazolium based ionic liquids. ${ }^{31}$ Additionally, the outcomes of our study provide detailed insights into the underlying binding mechanism with regard to a 'tunable co-solute' IL approach.

The molecular dynamics simulations were performed using the software package GROMACS (version 4.6.7) ${ }^{35,36}$ utilizing the non-polarizable CL\&P force field for EMIM and ACE, ${ }^{37-40}$ the OPLS/AA force field for proteins, ${ }^{41}$ which is compatible with the CL\&P force field ${ }^{37}$ and the TIP3P water model for the aqueous solution. ${ }^{42}$ The number of EMIM ACE pairs was $N_{\text {pairs }}=250$ which resulted in a concentration of $c=0.57 \mathrm{~mol} \mathrm{~L}^{-1}$. We used the original metadynamics method for the evaluation of the free energy landscapes at $298 \mathrm{~K}^{43-45}$ and chose the end-to-end distance $R_{\mathrm{e}}$ between the center-of-mass of the first and the last residue and the root-mean-square deviation (RMSD) with regard to the atomic positions of the energy-minimized initial PDB structure as collective variables. In order to analyze the binding behavior, we have evaluated the corresponding Kirkwood-Buff integrals ${ }^{46-48}$ to study the preferential binding coefficients. ${ }^{12,48}$ As a prerequisite, chain conformation effects have to be diminished which has been achieved by fixing the positions of the heavy $\mathrm{C}_{\alpha}$ atoms for the native folded peptide structure and a fully unfolded conformation. The unfolded structure has been obtained by high temperature simulations at $500 \mathrm{~K}$ which gave us also the chance to find appropriate collective variables for the metadynamics simulations to clearly distinguish between the folded and the unfolded conformation. ${ }^{49,50}$ Each production run of the restrained simulations had a length of $100 \mathrm{~ns}$ and was performed in an NpT ensemble at $298 \mathrm{~K}$ and 1 bar. More details on the simulation protocol can be found in the ESI. $\dagger$

We studied the binding behavior of the ionic liquid in terms of the radial distribution function and the preferential binding coefficient. The Kirkwood-Buff integral for computational purposes is given by

$$
G_{\alpha \beta}\left(r_{\mathrm{c}}\right) \approx 4 \pi \int_{0}^{r_{\mathrm{c}}} r^{2}\left[g_{\alpha \beta}^{N p T}(r)-1\right] \mathrm{d} r
$$

with the radial distribution function $g_{\alpha \beta}(r)$ between species $\alpha, \beta$ and the cut-off radius $r_{\mathrm{c}}{ }^{51-53}$ It has been often discussed that $r_{\mathrm{c}}$ has to be chosen such that all considered radial distribution functions show converged values at distances $r \geq r_{\mathrm{c}} \cdot{ }^{48,52}$ In the following, we omit $r_{\mathrm{c}}$ for the sake of clarity as an argument in the equations. With regard to conventional notations, the solvent is usually denoted as $\alpha, \beta=1$, the solute as 2 and the co-solute, here EMIM and ACE as 3. The preferential binding coefficient $\nu_{23}$ between the center-of-masses for all $\mathrm{C}_{\alpha}$ atoms of $2 \mathrm{~GB} 1$ and EMIM or ACE is given by

$$
\nu_{23}=\rho_{3}\left(G_{23}-G_{21}\right)
$$

where $\rho_{3}$ corresponds to the bulk number density of the ions. The effects of co-solutes on macromolecular conformations can be evaluated in terms of the chemical equilibrium constant $K=\pi_{\mathrm{u}} / \pi_{\mathrm{f}}$ where $\pi_{\mathrm{u}}$ and $\pi_{\mathrm{f}}$ denote the fraction of $2 \mathrm{~GB} 1$ in the unfolded (u) and the folded states (f). The relation ${ }^{48}$

$$
\left(\frac{\partial \ln K}{\partial \ln a_{3}}\right)=\Delta \nu_{23}=\nu_{23}^{\mathrm{u}}-\nu_{23}^{\mathrm{f}}
$$

finally connects the derivative of $\ln K$ with respect to the logarithmic chemical activity $\ln a_{3}$ of the ionic liquid with the preferential binding coefficients of the unfolded $\nu_{23}^{\mathrm{u}}$ and the folded conformations $\nu_{23}^{\mathrm{f}}$. The usage of the chemical activity $a_{3}$ instead of the IL concentration takes into account all deviations from an ideal solution and is a common approach. A positive value for $\Delta \nu_{23}$ indicates the shift of the chemical equilibrium towards the unfolded state whereas a negative value implies the stabilization of the folded state.

The results for the radial distribution functions between the center-of-masses of the $\mathrm{C}_{\alpha}$ atoms of 2GB1 and the EMIM, respectively, ACE ions (center-of-mass) are presented in Fig. 1. It can be clearly seen that the unfolded conformation of 2GB1 attracts more acetate ions than the folded state whereas the values for EMIM are nearly identical for both conformations. Moreover, the position of the highest EMIM peak at $1 \mathrm{~nm}$ in the radial distribution functions is roughly identical for the folded and the unfolded structure. Only slight deviations like the occurrence of a small EMIM peak at $0.7 \mathrm{~nm}$ around the unfolded conformation can be observed.

A significantly different behavior can be found for ACE. With regard to the folded structure, it can be observed that ACE is more or less excluded from the first hydration shells around the peptide at distances between 0.3 and $2.5 \mathrm{~nm}$. In contrast, the occurrence of a well pronounced acetate ion shell around the unfolded conformation within distances of 0.3 to $0.7 \mathrm{~nm}$ is obvious. Interestingly, a slight tendency to form EMIM ACE ion pairs around the unfolded structure at distances of $0.9 \mathrm{~nm}$ is evident. Noteworthily, a shell-like accumulation behavior of EMIM ACE around differently charged solutes has been also discussed in a recent publication. ${ }^{21}$ The formation of distinct ion shells is also revealed by the individual radial distribution functions between the peptide residues and the ionic liquid. A more detailed analysis with regard to the properties of the interactions validates that acetate ions strongly interact via electrostatic interactions with the peptide whereas the more
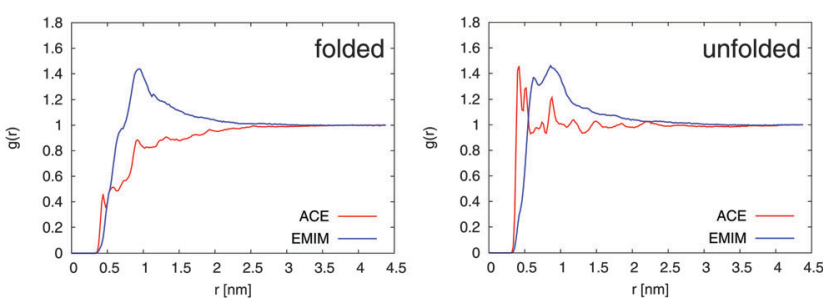

Fig. 1 Radial distribution function $g(r)$ between EMIM, respectively ACE (center-of-mass) and the $\mathrm{C}_{\alpha}$-atoms (center-of-mass) of 2GB1 for the folded (left side) and the unfolded conformation (right side). 
bulky EMIM cations induce stronger Lennard-Jones interactions. The corresponding analysis can be found in the ESI. $\dagger$

With regard to the Kirkwood-Buff integrals, the results for the preferential binding coefficient $\nu_{23}$ between the $\mathrm{C}_{\alpha}$-atoms and EMIM, respectively ACE ions for the folded and the unfolded peptide conformation are presented in Fig. 2. It becomes evident that EMIM ions strongly bind to both conformations of the peptide as it was discussed above due to positive and converged values for $\nu_{23}$ at large distances. Indeed, the preferential binding coefficients for EMIM are nearly identical for both structures which emphasizes the fact that the transfer free energies of EMIM with $\Delta F=-R T \nu_{23}$, where $R T$ denotes the thermal energy with the molar gas constant $R$, are also comparable. ${ }^{12}$ Thus, it can be assumed that a conformation dependent binding is absent and it can be concluded that the chemical equilibrium constant $K$ according to eqn (3) remains unchanged under the influence of EMIM. With regard to these findings, EMIM can be neither regarded as a denaturant nor a protectant of 2GB1 although it can be definitely considered as a chaotropic cosolute in terms of its binding behavior. ${ }^{6,34}$

In contrast to EMIM, acetate ions show a more pronounced preferential binding behavior to the unfolded conformation $\left(\nu_{23}^{\mathrm{u}}>0\right.$ and $\left.\nu_{23}^{\mathrm{f}}<0\right)$. Thus, a preferential exclusion mechanism can be observed for the folded conformation in contrast to a preferential binding behavior to the unfolded state. Indeed, the positive charge of the first EMIM layer attracts ACE anions that form a second layer around the native hairpin structure of the peptide. An increase of the solvent-accessible surface area due to unfolding promotes the formation of EMIM-ACE ion pairs at short distances around 2GB1 in addition to stronger electrostatic interactions with the peptide. More details on this mechanism which closely resembles the accumulation behavior of EMIM and ACE around differently charged and neutral spheres ${ }^{21}$ can be found in the ESI. $\dagger$ With regard to the corresponding influence on the chemical equilibrium constant according to eqn (3), it can be concluded that the presence of acetate ions induces the unfolding of the native peptide conformation. Indeed, these results are in good agreement with experimental findings, ${ }^{28,31}$ where it has been discussed that the choice of different anions in the presence of EMIM strongly influences the melting temperature of the protein ribonuclease A.

In addition to the preferential binding behavior, we have also performed metadynamics simulations to obtain the free
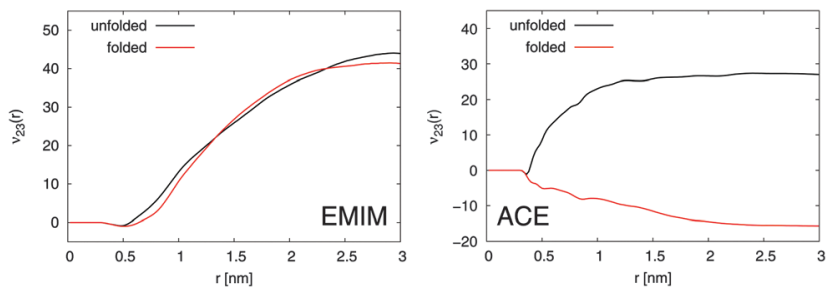

Fig. 2 Left: Preferential binding coefficient $\nu_{23}(r)$ between $C_{\alpha}$-atoms of 2GB1 and EMIM cations for the folded (red line) and the unfolded peptide conformation (black line). Right: Preferential binding coefficient between $\mathrm{C}_{\alpha}$-atoms of $2 \mathrm{~GB} 1$ and $\mathrm{ACE}$ anions for the folded (red line) and the unfolded (black line) peptide conformation. energy landscapes for the two peptide conformations in the presence of pure water and a 0.57 molar aqueous EMIM ACE solution. The corresponding results in pure water and in aqueous ionic liquid solution are presented in Fig. 3 and 4. It can be clearly seen that the most stable free energy minimum conformation in the presence of pure water is given by a $\beta$-hairpin structure at $R_{\mathrm{e}}=0.5-0.8 \mathrm{~nm}$ and a RMSD of $0.4-0.6 \mathrm{~nm}$. As a remark, a RMSD of zero corresponds to the reference structure of an energy minimized conformation without further equilibration which explains the occurrence of the large RMSD value for the local free energy minimum. The landscape clearly indicates the stability of a folded structure in a funnel-like global free energy minimum. Thus, the lowest free energy conformation is located in a very narrow region which is roughly $2 \mathrm{kcal} \mathrm{mol}^{-1}$ more stable than slightly distorted $\beta$-hairpin conformations. Moreover, it can be assumed that the relevant accessible phase space is restricted to free energy differences of $\Delta F \approx 5-8 \mathrm{kcal} \mathrm{mol}^{-1}$. These findings are in good agreement with previous results for 2GB1 in pure water although different collective variables for the evaluation of the corresponding free energy landscapes were used. $^{54,55}$

In contrast to these results, the lowest free energy conformation in the presence of the aqueous EMIM ACE solution is located at $R_{\mathrm{e}}=1.6 \mathrm{~nm}$ and a RMSD of $0.8 \mathrm{~nm}$ (Fig. 4). Thus, a significant broadening of the end-to-end distance compared with the stable folded conformation in pure water can be observed. Furthermore, it becomes obvious that the free energy basin in pure water which corresponds to the native $\beta$-hairpin structure in Fig. 3 vanishes in the presence of the ionic liquid. The resulting free energy landscapes verify that EMIM and ACE ions induce the denaturation of the native conformation in agreement with previous results. ${ }^{32}$ Moreover, it has to be noticed that the accessible phase space in

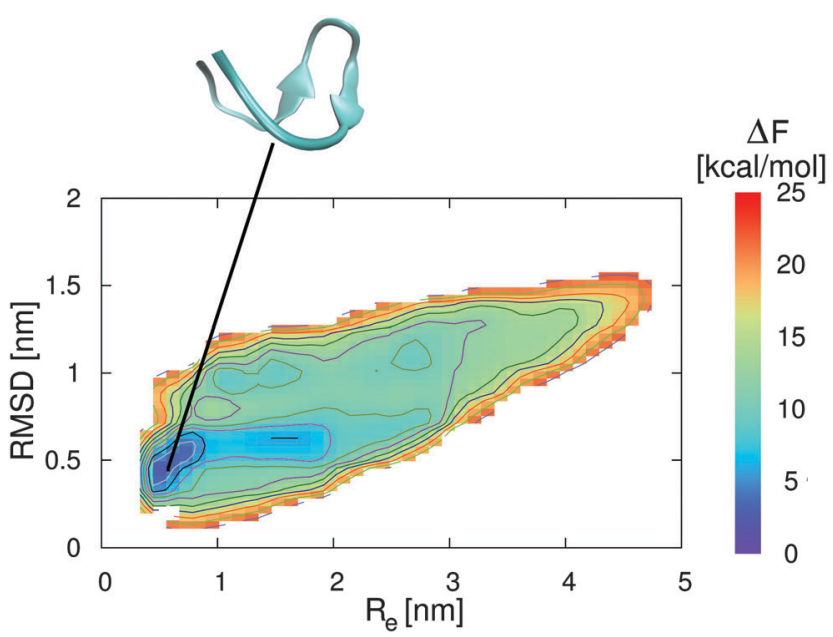

Fig. 3 Free energy landscape for 2GB1 in pure water. The collective variables are given by the end-to-end distance $R_{\mathrm{e}}$ between the first and the last residue of the strand (center-of-mass) and the root-mean square deviation (RMSD) to the energy minimized structure. The solid lines correspond to free energy differences of $2 \mathrm{kcal} \mathrm{mol}^{-1}$. The snapshot presented at the top corresponds to a native $\beta$-hairpin structure which represents the local free energy minimum conformation at the corresponding position as denoted by the black line. 


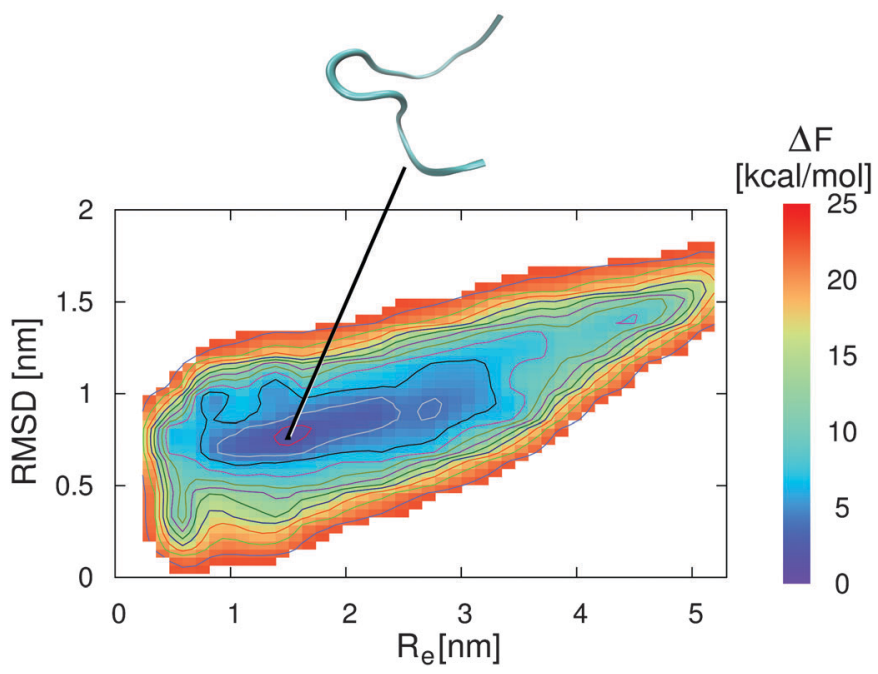

Fig. 4 Free energy landscape for $2 \mathrm{GB1}$ in an aqueous ionic liquid solution with a concentration of $c=0.57 \mathrm{~mol} \mathrm{~L}^{-1}$. The collective variables are given by the end-to-end distance $R_{\mathrm{e}}$ between the first and the last residue of the strand (center-of-mass) and the root-mean square deviation (RMSD) to the energy minimized structure. The solid lines correspond to free energy differences of $2 \mathrm{kcal} \mathrm{mol}^{-1}$. The snapshot presented at the top corresponds to an unfolded peptide structure which represents the local free energy minimum conformation at the corresponding position as denoted by the black line.

the presence and absence of EMIM ACE for all values of $\Delta F \leq$ $25 \mathrm{kcal} \mathrm{mol}^{-1}$ remains roughly unchanged. With regard to the results, it can be concluded that the ionic liquid induces a displacement of the global free energy minimum in terms of the native $\beta$-hairpin state to a new location as compared with the pure water results.

In summary, we have studied the influence of an ionic liquid, namely 1-ethyl-3-methylimidazolium acetate, in an aqueous solution on the stability of a peptide fragment from protein $\mathrm{G}$ by all-atom molecular dynamics simulations. With regard to the Kirkwood-Buff theory, we were able to study the ionic preferential binding behavior. Our results indicate large and nearly identical preferential binding parameters of EMIM to the folded and the unfolded peptide structure whereas ACE exclusively binds to the unfolded conformation. These results clearly indicate that the presence of acetate anions can be regarded as the main driving force for the denaturation of the hairpin structure. Indeed, the presence of large cations like EMIM, which form a first layer around the peptide due to strong Lennard-Jones interactions, is mandatory for the accumulation of the acetate ions. Hereby, EMIM cations of the first layer attract the oppositely charged ACE ions due to electrostatic interactions. The preferential binding of ACE at shorter distances is then facilitated by an unfolding of the native structure. The results of the presented metadynamics simulations finally validate the energetic destabilization of the native $\beta$-hairpin structure in the presence of an aqueous EMIM ACE solution. With regard to the previous discussion and the binding properties, we conclude that EMIM ACE behaves like a denaturant.

Indeed, it can be assumed that the presence of two ion species gives rise to new functionalities in terms of 'tunable co-solutes'.
With regard to this assumption, it has been found in ref. 32 and discussed in ref. 28 that the exchange of the imidazolium cation with protic cations like choline would result in a significant increase of the protein melting temperature. Therefore, ionic liquids might be regarded as potential candidates to overcome the limitations of common denaturants in terms of tunable functionalities.

We greatly acknowledge the helpful discussions with Anand Narayanan Krishnamoorthy, Ewa Anna Oprzeska-Zingrebe, Julian Michalowsky, Samantha Micciulla, Jan Heyda, Francisco Rodriguez-Ropero, Nico van der Vegt and Pavel Jungwirth. The authors thank the Deutsche Forschungsgemeinschaft through the cluster of excellence initiative 'Simulation Technology' (EXC 310), the collaborative research centers SFB 716 and TRR 61 and the federal ministry of research and education (Förderkennzeichen: 03X4631N) for financial support.

\section{References}

1 P. H. Yancey, J. Exp. Biol., 2005, 208, 2819-2830.

2 D. R. Canchi and A. E. Garca, Annu. Rev. Phys. Chem., 2013, 64, 273-293.

3 D. Harries and J. Rösgen, Methods Cell Biol., 2008, 84, 679-735.

4 R. Politi and D. Harries, Chem. Commun., 2010, 46, 6449-6451.

5 K. D. Collins, Biophys. J., 1997, 72, 65-76.

6 K. D. Collins, Methods, 2004, 34, 300-311.

7 K. D. Collins, G. W. Neilson and J. E. Enderby, Biophys. Chem., 2007, 128, 95-104.

8 Y. Zhang and P. S. Cremer, Annu. Rev. Phys. Chem., 2010, 61, 63-83.

9 J. Smiatek, R. K. Harishchandra, O. Rubner, H.-J. Galla and A. Heuer, Biophys. Chem., 2012, 160, 62-68.

10 J. Smiatek, R. K. Harishchandra, H.-J. Galla and A. Heuer, Biophys. Chem., 2013, 180, 102-109.

11 A. Narayanan Krishnamoorthy, C. Holm and J. Smiatek, J. Phys. Chem. B, 2014, 118, 11613-11621.

12 J. Smiatek, J. Phys. Chem. B, 2014, 118, 771-782.

13 T. Yasuda and M. Watanabe, MRS Bull., 2013, 38, 560-566.

14 N. M. Micaêlo and C. M. Soares, J. Phys. Chem. B, 2008, 112, 2566-2572.

15 P. Wasserscheid and W. Keim, Angew. Chem., 2000, 39, 3772-3789.

16 A. Matic and B. Scrosati, MRS Bull., 2013, 38, 533-537.

17 O. Borodin, G. D. Smith and W. Henderson, J. Phys. Chem. B, 2006, 110, 16879-16886.

18 O. Borodin, J. Phys. Chem. B, 2009, 113, 12353-12357.

19 Z. Li, G. D. Smith and D. Bedrov, J. Phys. Chem. B, 2012, 116, 12801-12809.

20 V. Lesch, S. Jeremias, A. Moretti, S. Passerini, A. Heuer and O. Borodin, J. Phys. Chem. B, 2014, 118, 7367-7375.

21 V. Lesch, A. Heuer, C. Holm and J. Smiatek, Phys. Chem. Chem. Phys., 2015, 17, 8480-8490.

22 K. R. Seddon, J. Chem. Technol. Biotechnol., 1997, 68, 351-356.

23 D. Bedrov, O. Borodin, Z. Li and G. D. Smith, J. Phys. Chem. B, 2010, 114, 4984-4997. 
24 M. Klähn, G. S. Lim, A. Seduraman and P. Wu, Phys. Chem. Chem. Phys., 2011, 13, 1649-1662.

25 M. Haberler, C. Schröder and O. Steinhauser, Phys. Chem. Chem. Phys., 2011, 13, 6955-6969.

26 M. Haberler, C. Schröder and O. Steinhauser, J. Chem. Theory Comput., 2012, 8, 3911-3928.

27 M. Haberler and O. Steinhauser, Phys. Chem. Chem. Phys., 2012, 13, 17994-18004.

28 R. Patel, M. Kumari and A. B. Khan, Appl. Biochem. Biotechnol., 2014, 172, 3701-3720.

29 N. Byrne and C. A. Angell, J. Mol. Biol., 2008, 378, 707-714.

30 A. Kumar and P. Venkatesu, RSC Adv., 2013, 3, 362-367.

31 D. Constantinescu, H. Weingärtner and C. Herrmann, Angew. Chem., Int. Ed., 2007, 46, 8887-8889.

32 H. Weingärtner, C. Cabrele and C. Herrmann, Phys. Chem. Chem. Phys., 2012, 14, 415-426.

33 R. Hayes, G. G. Warr and R. Atkin, Chem. Rev., 2015, 115, 6357-6426.

34 P. Lo Nostro and B. W. Ninham, Chem. Rev., 2012, 112, 2286-2322.

35 D. van der Spoel, E. Lindahl, B. Hess, G. Groenhof, A. E. Mark and H. J. C. Berendsen, J. Comput. Chem., 2005, 26, 1701-1718.

36 S. Pronk, S. Páll, R. Schulz, P. Larsson, P. Bjelkmar, R. Apostolov, M. R. Shirts, J. C. Smith, P. M. Kasson, D. van der Spoel, B. Hess and E. Lindahl, Bioinformatics, 2013, 29, 845-854.

37 J. N. Canongia Lopes, J. Deschamps and A. A. H. Padua, J. Phys. Chem. B, 2004, 108, 2038-2047.

38 J. N. Canongia Lopes and A. A. H. Padua, J. Phys. Chem. B, 2006, 110, 19586-19592.
39 J. N. Canongia Lopes and A. A. H. Padua, J. Phys. Chem. B, 2004, 108, 16893-16898.

40 J. N. Canongia Lopes, A. A. H. Padua and K. Shimizu, J. Phys. Chem. B, 2008, 112, 5039-5046.

41 G. A. Kaminski, R. A. Friesner, J. Tirado-Rives and W. L. Jorgensen, J. Phys. Chem. B, 2001, 105, 6474-6487.

42 W. L. Jorgensen, J. Chandrasekhar, J. D. Madura, R. W. Impey and M. L. Klein, J. Chem. Phys., 1983, 79, 926-935.

43 A. Laio and M. Parrinello, Proc. Natl. Acad. Sci. U. S. A., 2002, 99, 12562-12566.

44 A. Laio and F. L. Gervasio, Rep. Prog. Phys., 2008, 71, 126601.

45 J. Smiatek and A. Heuer, J. Comput. Chem., 2011, 32, 2084-2096.

46 J. G. Kirkwood and F. P. Buff, J. Chem. Phys., 1951, 19, 774-777.

47 P. E. Smith, Biophys. J., 2006, 91, 849-856.

48 V. Pierce, M. Kang, M. Aburi, S. Weerasinghe and P. E. Smith, Cell Biochem. Biophys., 2008, 50, 1-22.

49 J. Smiatek, D. Janssen-Müller, R. Friedrich and A. Heuer, Physica A, 2014, 394, 136-144.

50 J. Smiatek, D. Liu and A. Heuer, Curr. Phys. Chem., 2012, 2, 115-123.

51 P. E. Smith, J. Phys. Chem. B, 1999, 103, 525-534.

52 S. Weerasinghe and P. E. Smith, J. Phys. Chem. B, 2003, 107, 3891-3898.

53 J. Smiatek, A. Wohlfarth and C. Holm, New J. Phys., 2014, 16, 025001.

54 R. Zhou, B. J. Berne and R. Germain, Proc. Natl. Acad. Sci. U. S. A., 2001, 98, 14931-14936.

55 G. Bussi, F. L. Gervasio, A. Laio and M. Parrinello, J. Am. Chem. Soc., 2006, 128, 13435-13441. 